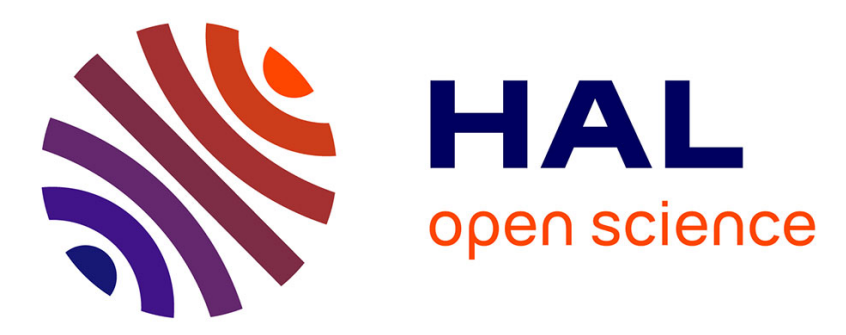

\title{
NMR Study of Water Distribution inside Tomato Cells: Effects of Water Stress
}

\author{
M. Musse, M. Cambert, F. Mariette
}

\section{To cite this version:}

M. Musse, M. Cambert, F. Mariette. NMR Study of Water Distribution inside Tomato Cells: Effects of Water Stress. Applied Magnetic Resonance, 2010, 38 (4), p. 455 - p. 469. 10.1007/s00723-0100139-7 . hal-00581264

\section{HAL Id: hal-00581264 https://hal.science/hal-00581264}

Submitted on 30 Mar 2011

HAL is a multi-disciplinary open access archive for the deposit and dissemination of scientific research documents, whether they are published or not. The documents may come from teaching and research institutions in France or abroad, or from public or private research centers.
L'archive ouverte pluridisciplinaire HAL, est destinée au dépôt et à la diffusion de documents scientifiques de niveau recherche, publiés ou non, émanant des établissements d'enseignement et de recherche français ou étrangers, des laboratoires publics ou privés. 
Author-produced version of the article published in Applied Magnetic Resonance, 2010, 38, 4. 455-459.

Original publication available at www.springerkink.com - doi: 10.1007/s00723-010-0139-7

NMR study of water distribution inside tomato cells: Effects of water stress

Maja Musse*, Mireille Cambert, François Mariette

Cemagref, UR TERE, 17 avenue de Cucillé, CS 64427, F-35044 Rennes France.

Université européenne de Bretagne, France.

* Corresponding author

Tel: +332234821 79; Fax: +33223482115

E-mail address: maja.musse@cemagref.fr

\section{ABSTRACT}

Tomato pericarp tissue was studied by low field nuclear magnetic resonance (NMR) Relaxometry. Two kinds of experiment were performed in order to investigate the correlation between multi-exponential NMR relaxation and the subcellular compartments. Longitudinal $\left(T_{1}\right)$ versus transverse $\left(T_{2}\right)$ relaxation times were first measured on fresh samples and then the transverse relaxation time was measured on samples exposed to water stress. Four signal components were found in all experiments. The results showed that all signal components corresponded to the water in different cell compartments, and that no signal from non-exchangeable protons was present. Moreover, we demonstrated that NMR relaxation is suitable for the continuous monitoring of water rebalancing between subcellular compartments of plant tissues.

Key words: NMR; $\mathrm{T}_{1} ; \mathrm{T}_{2}$; plant cells; cell compartments

\section{INTRODUCTION}

NMR relaxation time measurements have been used in several studies to investigate vegetal cells $[1,2]$. The relaxation signals from vegetal cells have generally been described by a multi-exponential behaviour reflecting different water compartments. The longitudinal $\left(T_{1}\right)$ and transverse relaxation $\left(T_{2}\right)$ times are known to be related to the water status in the 
compartments, i. e. water content, water mobility and interactions between water and macromolecules.

Although NMR has been shown to be able to provide useful information about plant cells, the interpretation of the results is not always straightforward. The number of NMR signal components undoubtedly depends on the plant tissue, but it seems also be related to the measurement protocol and the fitting method, even when the classical CRMG sequence is used. For example, a single component of $\mathrm{T}_{2}$ relaxation decay was reported in the whole tomato fruit by Tu et al. [3], while four components were found in the tomato pericarp by Duval et al. [4], Marigheto [5] as well as in our previous studies [6, 7]. Furthermore, the precise assignment of the NMR signal components to the specific subcellular compartments is still a subject of debate. Only a few investigations have been focused on the attribution of the NMR signal components by probing water compartments. Three components of the NMR signal of apple cells have been assigned to the vacuole, cytoplasm and wall/extracellular space by following the uptake of $\mathrm{Mn}^{2+}$ in the tissue [2]. The same component attributions were also proposed by Hills and Remigereau [8]. More recently, four components in $T_{2}$ were found in apple cells and were assigned to the vacuole, cytoplasm, extracellular space and cell wall $[9,10]$. However, this attribution and distribution does not necessarily hold for all types of plant tissue. For example, Mariette et al. [11] extracted four components in potatoes, and the component with the shortest $T_{2}$ was attributed to the non-exchangeable starch protons, while other components were attributed to the water protons from the different cell compartments (vacuole, cytoplasm and cell wall). Two components have been extracted in carrot parenchymal tissue and they were attributed to the vacuole and cell wall/extracellular space compartments [8]. In the latter study, a numerical cell model describing cell compartments was used to explain the NMR signal. Furfaro et al. [12] recently proposed another interpretation of the $T_{2}$ relaxation in carrot xylem and phloem tissues, assuming a contribution of non-exchangeable macromolecular protons. From a $T_{1}-T_{2}$ relaxation experiment performed at $23.4 \mathrm{MHz}$ they identified three components and attributed them to cell water and to non-exchangeable protons from pectin and cellulose. For the tomato $[4,6]$, 
the components were assigned to the vacuole, cytoplasm, cell wall and non-exchangeable solute protons by association with the potato, but no experiment was performed to validate this hypothesis. Most recently, Marigheto et al [5] met difficulties with the assignment of the four relaxation time components in green tomato pericarp. Indeed, two interpretations were proposed according to the experiments. The first interpretation associated the components with water in the vacuole, extra-cellular water, cytoplasm and the more rigid components of the cell wall and the second one associated the components with water in the vacuole, cytoplasm/extra-cellular compartments, cell wall and water inside starch granules. Further investigations are therefore necessary to make progress in the attribution of NMR signal components to the subcellular compartments for different plant types. This step is essential to provide accurate interpretation of the measurements and thus make it possible to use all the potential of NMR techniques in plant research.

Quantitative information about subcellular compartments via relaxation time measurements can be particularly relevant for investigation of different processes undergone by plants, such as ripening, osmotic dehydration and water stress. The macroscopic transport of water through tissues is mainly controlled by the microscopic distribution of water and air on a cellular and subcellular distance scale and by the extent of membrane permeability. It is thus important to investigate the subcellular water distribution that occurs during plant transformations in order to understand macroscopic water transfer. This is very important for use in environment-stressed plant research and can be applied for assisting agricultural practices. Such studies have been performed on different types of plant for different processes. For example, changes in subcellular water distribution during ripening have been studied in tomato [5, 7] and banana [13] fruits. Iwaya-Inoue et al. [14] monitored post-harvest sweet potato tubers by NMR and identified changes in water status in subcellular compartments produced by water stress. Capitani et al. [15] used a portable unilateral NMR instrument to detect in field conditions the water status of leaves of herbaceous crops, mesophyllous trees, and natural Mediterranean vegetation. 
The aim of the study presented here was to provide greater understanding of NMR relaxation in tomato pericarp tissue in order to improve the knowledge about the attribution of NMR signal components to subcellular compartments and to investigate the response of specific cell compartments to water stress. Two kinds of experiment were performed. Firstly, two dimensional $T_{1}-T_{2}$ relaxation correlation experiments were carried out on fresh pericarp samples, allowing the identification of the molecular species based on the $T_{1} / T_{2}$ ratio [16]. This experiment made it possible to determine whether all components of the multiexponential NMR signals corresponded to subcellular water compartments, and particularly whether the component characterised by the shortest $T_{2}$ relaxation time corresponded to water or to non-exchangeable protons from non-aqueous molecules. Pericarp samples were then exposed to water stress and the NMR measurements were performed continuously during water rebalancing. Tomatoes were considered at two physiological stages by using green and red ripe tomato samples.

\section{MATERIALS AND METHODS}

\section{Plant Material}

Tomatoes (Lycopersicon esculentum Mill. var Admiro) provided by the CTIFL (Centre Technique Interprofessionel des Fruits et Légumes, France) were used in this study. Fruits for $T_{2}, T_{1}$ and $T_{1}-T_{2}$ measurements were picked at the late green stage (tomato colour code 3-4, CTIFL, France) and left to ripen in a constantly aerated ripening chamber under controlled conditions $\left(18^{\circ} \mathrm{C}\right.$ and $\left.55 \% \mathrm{RH}\right)$ for eight days. Five measurements were performed on samples taken from individual fruits. In the water stress experiment, a freshly picked green tomato (colour code 3-4) was compared to a freshly picked red tomato (colour code 7). Three measurements were performed on samples taken from the green tomato, and three on samples from the red tomato.

For each experiment, samples were prepared from the outer pericarp as follows: a one centimeter thick slice was cut in the equatorial region perpendicular to the pedicle axis, and 
cylinders $(0.8 \mathrm{~cm}$ in diameter) were cut into the tissue. Samples were wiped to remove water from the broken cells and then placed in NMR tubes and closed with a cap.

After NMR experiments, the water content of samples was estimated by measuring differences in weight after drying in an oven at $103^{\circ} \mathrm{C}$ for 24 hours.

A partial drying protocol used for the water stress experiment that significantly decreases the water content without cell deterioration was set up prior to measurements. In this protocol, cylindrical tissue samples were dried under different temperatures and pressures and cells were observed using a microscope (Nikon Eclipse 80i) equipped with a digital camera (DXM 1200c). The optimum protocol was found to be drying of samples in a vacuum oven at $30^{\circ} \mathrm{C}$ and $50 \mathrm{mbar}$ for $45 \mathrm{~min}$. Samples were placed in caps during drying.

\section{Methods}

NMR Relaxometry measurements were performed on a $20 \mathrm{MHz}(0.47 \mathrm{~T})$ spectrometer (Minispec PC-120, Bruker, Karlsruhe, Germany) equipped with a thermostatted probe at $18^{\circ} \mathrm{C}$

Separate $T_{2}$ and $T_{1}$ measurements were performed in addition to the $T_{1}-T_{2}$ measurements for comparison purposes. The Carr-Purcell-Meiboom-Gill (CPMG) sequence was used for $T_{2}$ measurement, $90^{\circ}-180^{\circ}$ pulse spacing was $0.1 \mathrm{~ms}$ and 5000 even echoes were recorded. The duration of the $90^{\circ}$ pulse was $18 \mu$ s. Data were averaged over eight acquisitions and the recovery delay was $12 \mathrm{~s} . T_{1}$ was measured using a saturation recovery sequence (SR). One hundred points were acquired from $30 \mathrm{~ms}$ to $12 \mathrm{~s}$. The saturation-recovery times ( $\left.\mathrm{t}_{\mathrm{SR}}\right)$ were spaced according to :

$$
\mathrm{t}_{\mathrm{SR}}(\mathrm{i})=\mathrm{t}_{0}+\frac{(\mathrm{i}+1) \mathrm{x}\left(\mathrm{t}_{\mathrm{f}}-\mathrm{t}_{0}\right)}{(\mathrm{n}-1)^{3}}
$$

with $t_{0}$ and $t_{f}$ the first and the last recovery time, respectively, and $n$ the number of recovery time values used.

The relaxation curves were fitted by Scilab software according to the maximum entropy method (MEM) [17], which provides a continuous distribution of relaxation components 
without any assumption concerning their number, and the Levenberg-Marquardt algorithm which will fit the data to the multi term exponential model. In the latter method, the number of terms that best described the relaxation curve was determined by examining the residual plots and the values of the coefficient of determination. $T_{2}$ relaxation curves were fitted using the Levenberg-Marquardt algorithm according to the equation:

$\mathrm{I}_{\mathrm{T} 2}(\mathrm{t})=\sum_{\mathrm{i}} \mathrm{I}_{0 \mathrm{i}} \exp \left(-\mathrm{t} / \mathrm{T}_{2, \mathrm{i}}\right)$

where $I_{0 i}$ is the intensity of the $i_{t h}$ exponential at the equilibrium state and $T_{2, i}$ the characteristic transverse relaxation time for the $\mathrm{i}_{\text {th }}$ exponential. $\mathrm{T}_{1}$ relaxation curves were fitted using the equation:

$\mathrm{I}_{\mathrm{T} 1}(\mathrm{t})=\sum_{\mathrm{i}} \mathrm{I}_{0 \mathrm{i}}\left(1-\alpha \exp \left(-\mathrm{t} / \mathrm{T}_{1, \mathrm{i}}\right)\right)$

where $\mathrm{I}_{0 \mathrm{i}}$ is the intensity of the $\mathrm{i}_{\mathrm{th}}$ exponential at the equilibrium state and $T_{1, i}$ the characteristic longitudinal relaxation time for the $\mathrm{i}_{\mathrm{th}}$ exponential. $\alpha$ is a parameter that takes into account the $90^{\circ} \mathrm{RF}$ pulse imperfection, its value is being generally equal to one for a perfect $90^{\circ} \mathrm{RF}$ pulse .

A $90^{\circ}$ RF pulse was added before the CPMG sequence to provide a sequence for $2 D T_{1}-T_{2}$ correlation measurements (SR-CPMG). The experiment was performed by varying $t_{\mathrm{SR}}$ and consequently the $T_{1}$-weighting of the CPMG decay. We used 50 values between $30 \mathrm{~ms}$ and $12 \mathrm{~s}$ for $\mathrm{t}_{\mathrm{SR}}$.

Assuming that each compartment is characterised by one $T_{2}$ and one $T_{1}$ value, the signal amplitude is given by the following equation:

$$
\mathrm{I}\left(t_{C P M G}, t_{S R}\right)=\sum_{\mathrm{i}} \mathrm{I}_{0, \mathrm{i}} \exp \left(-\mathrm{t}_{\mathrm{CPMG}} / \mathrm{T}_{2 \mathrm{i}}\right)\left(1-\alpha \exp \left(-\mathrm{t}_{\mathrm{SR}} / \mathrm{T}_{1 \mathrm{i}}\right)\right)
$$

The SR-CPMG 2D signals were fitted by a two-step method. Firstly each CPMG curve was fitted by a multi-exponential model in order to extract the $T_{2}$ values and the associated intensities. The intensity of each $\mathrm{T}_{2}$ component was then plotted against $t_{\mathrm{SR}}$ and the $\mathrm{SR}$ decay curve was fitted with equation 3. 
In the experiment to study water rebalancing inside cell compartments after subjection to water stress, $T_{2}$ relaxation decay was measured on the samples before applying the stress, at the end of the stress application and every three minutes for three hours. The measurements were performed on samples taken from the green and red tomatos.

In addition to the dynamic measurements, the amplitudes of the NMR signals from the outer pericarp before and after subjection to water stress were compared to the amplitude of the signal expected from distilled water. The signals expected from water were calculated for each sample as the product of its mass, its water content and the signal of the distilled water per unit mass. Three samples of the outer pericarp of the red tomatoes were used for this experiment. The aim was to investigate whether all components of the multi-exponential NMR signals could be assigned to water in cell compartments.

\section{RESULTS AND DISCUSSION}

\section{$T_{2}, T_{1}$ and $T_{1}-T_{2}$ Correlation Measurements}

Relaxation decays of the CPMG sequence were well described by four separate components, and decays of the SR sequence by two components, independently of whether the adjustment method was continuous (MEM) or discrete (Levenberg-Marquardt algorithm). An example of transverse and longitudinal relaxation distribution is given in Fig. 1 and the results obtained by the discrete adjustment method are summarized in Table1 (1D). The relaxation times and relative signal amplitudes were in excellent agreement with our previous results performed on other tomato samples [6].

Component 4 with the longest $T_{2}$ of $1528 \mathrm{~ms}$ and $68 \%$ relative signal intensity, was associated with the water in the vacuole, because of its high relative intensity and its long relaxation time. In fact, the vacuole contains about $50-80 \%$ of the cell water according to tissue [18]. The attribution of the other three components is less obvious. However, it can be assumed that component 3 , with $T_{2}=551 \mathrm{~ms}$ and a relative signal intensity of $20 \%$, was associated with the cytoplasm, while component 2 , with $T_{2}=134 \mathrm{~ms}$ and a relative signal intensity of $9 \%$, was assigned to the cell wall. The interpretation of components 2 and 3 is not 
straightforward at this stage as the cell wall of some vegetable tissues may contain a greater water fraction than cytoplasm [18]. The interpretations given in the literature $[4,5,10]$ differ for component 1 , characterised by the lowest $T_{2}$ and the lowest relative intensity, and no experimental study to our knowledge has attributed it to a specific cell compartment.

$\mathrm{T}_{2}$ relaxation curves recorded in the SR-CPMG experiments with different $t_{\mathrm{SR}}$ delays were fitted independently, according to the four-exponential transverse relaxation decay given by equation 2 for $i=4$, and $T_{2}$ values are plotted in Fig. 2. Variation in $T_{2}$ was relatively low: about $28 \%$ for the component $1,19 \%$ for component $2,12 \%$ for component 3 and $4 \%$ for component 4 . For the components 1 and 2 variation in $T_{2}$ was calculated for $t_{S R}<3000$ ms, and for the components 3 and 4 variation in $T_{2}$ was calculated for $t_{\mathrm{SR}}<9000 \mathrm{~ms}$. In the case of components 3 and 4 , the first seven points were eliminated from the statistics as aberrant, due to the low signal to noise ratio of the NMR signal for the very short $t_{\mathrm{SR}}$ values, and consequently to less accurate fitting. The $T_{2}$ variation for each $t_{S R}$ value was in agreement with the standard deviation of the $T_{2}$ parameter. It can be concluded that the $T_{2}$ value did not depend on $t_{\mathrm{SR}}$, indicating that each signal component corresponded to a distinct proton population.

After fitting the CPMG signal, the intensities of each component were plotted according to $t_{S R}$ to provide a $T_{1}$ relaxation decay for each $T_{2}$ component. $T_{1}$ was calculated for each component by fitting with the mono-exponential function given by equation 3 for $\mathrm{i}=1$ (Fig. 3 ). The $T_{2}$ and $T_{1}$ values obtained with the SR-CPMG sequence are summarized in Table 1 (2D) and compared to the values obtained with the independent $T_{2}$ and $T_{1}$ measurements. $T_{2}$ values obtained from the two methods matched well, whereas slight differences were observed in $T_{1}$ measurements. These differences can be explained by the relatively low signal to noise ratio of the SR-CPMG sequence compared to the 1D SR measurements, and consequently to the less accurate fitting procedure. The SR-CPMG experiment confirmed that the relaxation decay of the pericarp tissue can be described for both $T_{1}$ and $T_{2}$ by four relaxation components. Despite we used a two-step method for the fitting of the $T_{1}-T_{2}$ data, a 
number of relaxation component is in agreement with the results from Marigheto [5] obtained with a 2D inverse-Laplace transformation.

Fig. 4 shows the $T_{1}-T_{2}$ distribution map. The $T_{1} / T_{2}$ ratio was constant, which demonstrated that the relaxation mechanisms involved were the same in all subcellular compartments [19]. It was greater than 1 (shown as a dashed line in the $T_{1}-T_{2}$ plot) as expected for biological tissues, because of the presence of chemical exchanges. Thus all components corresponded to water protons, and no other non-exchangeable protons were detected in this experiment. Regarding these results and the results from our previous measurements which showed that the component 1 was present throughout the maturation process [7], the component with the shortest transverse relaxation time could correspond to water in organelles, such as plastids.

\section{Water stress experiment}

Comparison of the pure water NMR signal and the NMR signals from the tomato pericarp before and after applying water stress.

The amplitudes of the signals from the fresh and dried tomatoes were compared with the expected signals from the pure water calculated for the same mass and water content as the tomato samples. The results are summarised in Table 2 . The difference between the measured signal intensity $\left(I_{M}\right)$ and the expected signal intensity $\left(I_{E}\right)$ was insignificant for both the fresh and the dried tomatoes. Compared to what would have occurred if the nonexchangeable protons had contributed to the signal, the expected signals from the pure water were always slightly lower than the measured signals. The small difference between the signals could be explained by the slight contribution of the exchangeable protons to the water signal. These results demonstrated that only the water signal was detected and that all components of the NMR signal corresponded to water in different cell compartments. The hypothesis of non-exchangeable protons contributing to the NMR signal was thus no longer valid, the signal of other proton-containing compounds decayed too quickly for our 
measurements. The results of this experiment were in agreement with the results in the previous section.

\section{Rebalancing of water in tomato tissue after applying water stress}

Fig. 5 shows the variations in NMR signal intensity (CPMG) according to time before and after applying water stress for the green (a) and the red tomatoes (b). The intensity was normalised by the initial sample mass in order to make a direct comparison between the experiments. Four components were detected in the NMR signal in all measurements, indicating that the internal cell organization was not affected by water stress and that water was distributed between the same cell compartments. It should be noted that the observation under the microscope showed that the cell walls were not damaged by drying.

The curves shown in Fig 5 can be divided into two parts: the first part (the first (i) and second (ii) points) related to the effects of water stress on the cells and second part (from point (ii) to the end (point (iii)) describing the water transfer between the subcellular compartments during the three-hour rebalancing period. The sum of the signal intensities of the four components did not significantly differ between the green and the red tomatoes. For the first point (fresh samples) it was 20.55 and 20.49 for the green and the red tomatoes, respectively. After applying the water stress, the sum of signal intensities was constant during the rebalancing period (i.e. $10.79 \pm 0.01$ and $10.27 \pm 0.06$ for the green and red tomatoes, respectively), demonstrating that no dehydration occurred during the three hours. However, the water distribution between cell compartments of the fresh samples (first point of the curve, Fig. 5) differed between different ripening stages. The relative signal intensities of each cell compartment at different points are given in Table 3. For the fresh green tomato, the water in the vacuole corresponded to about $70 \pm 5 \%$ of the total amount of water, compared to $64 \pm 5 \%$ for the fresh red tomato while the relative intensity of component 2 of the fresh samples was lower for the green than for the red tomato. These results are consistent with the results of our previous study [7]. 
After applying water stress for both the green and red tomatoes, there was a decrease in the signal intensity of components 4 and 3, corresponding to the vacuole and the cytoplasm, respectively. The intensity of component 2 increased slightly for the green tomato and decreased for the red tomato, whereas the intensity of component 1 increased for both tomatoes. Hills et al. [8] demonstrated that air-drying of apple parenchyma tissues resulted in loss of water from the vacuole, with very little change in the water content of the other compartments. However, the protocol used in their experiments included a 24-hour storage period between the drying step and the NMR measurements. This storage period was used to allow water equilibration between cell compartments. Our experiments demonstrated that in the absence of an equilibrium period both compartments (vacuole and cytoplasm) were affected by water stress. The difference in behaviour of component 2 between the green and the red tomatoes could indicate the attribution to the cell wall as the ability of the cell wall to retain water is influenced by changes in cell wall polysaccharides during fruit ripening. Further studies are however required to verify this hypothesis.

In the second part of the curves (describing the rebalancing period), the intensity of component 4 decreased significantly and the intensity of component 3 increased for both the green and red tomatoes. The intensity of component 2 increased significantly for the green tomato, while it remained almost constant for the red tomato. Component 1 was stable for both tomatoes. It should be noted here that component 1 was present in this experiment for both green and red tomatoes according to our previous study [7].

The NMR experiment reflected here one of the roles of the vacuole, i.e. to supply water to other cell compartments when a cell is submitted to stress. The vacuole therefore continued to transfer water to the cytoplasm and the cell wall, thus ensuring cell endurance. It can be computed form the data presented in Fig. 5 that in the case of the green tomato the relative signal intensity loss (reflecting relative amount of water loss) produced by water stress was lower for component 4 than for component $3(48 \%$ and $72 \%$, respectively) while it was almost equal for these two components for the red tomato (53\% and $57 \%$, respectively), 
demonstrating that the response of specific cell compartments to water stress depends on the stage of maturation of the fruit.

On Fig $6 \mathrm{a}$ and $\mathrm{b}, \mathrm{T}_{2}$ is plotted according to time before and after applying water stress for green and red tomatoes, respectively. $T_{2}$ remained unchanged for the green tomato between the first two points (corresponding to time before and after applying water stress) for components 4 and 3, while it decreased slightly for component 2, and increased slightly for component 1 . In the red tomato, $T_{2}$ decreased between the first two points for all components, except for component 4.

The $T_{2}$ of water is known to be related to water content and membrane permeability [20]. Thus for constant membrane permeability a decrease in the water content should induce a decrease in $T_{2}$. Moreover, the $T_{2}$ of water is sensitive to the structure and the mobility of the surrounding molecules through chemical exchange and dipole-dipole interactions. Therefore a change in the non-aqueous molecular mobility should induce a change in the $T_{2}$ of water. According to these two mechanisms, we expected a decrease in $T_{2}$ for components 4 and 3 , which exhibited the greatest signal loss. However, the $T_{2}$ of these components remained constant after applying water stress for the green tomato, whereas it decreased only slightly for component 3 of the red tomato. It should be noted here that lack of sensitivity of the $T_{2}$ to the water loss was also reported by Hills et al. [8]. In fact, in apple cells the $T_{2}$ of the vacuole remained constant for below $45 \%$ of the mass loss, which corresponds to the mass loss which occurred in our experiment. This could therefore mean that $T_{2}$ is not sensitive to relatively low water loss (in the case of the tomato pericarp, $50 \%$ of the mass loss corresponds to $5 \%$ of water content loss). The slight variation observed can be explained by changes in the structure of molecules dispersed in the water phase induced by the treatment. The latter phenomenon could explain the differences observed between the green and red tomatoes. Another hypothesis is that lack of significant changes in $T_{2}$ is due to a decrease in the permeability induced by the treatment.

During the rebalancing period the correlation between the $T_{2}$ and the NMR signal amplitude observed was as expected. For the green tomato, both the $T_{2}$ and the signal intensity of the 
vacuole decreased. For the red tomato, the $T_{2}$ of the vacuole decreased for about 20 minutes and after that it remained mostly constant, corresponding to the decrease in signal intensity which was marked at the beginning of the post-stress period. The $T_{2}$ was constant for the other components.

\section{CONCLUSION}

The aim of this study was to improve understanding of the multi-exponential NMR relaxation in the tomato fruit pericarp and of the link between signal components and the cell compartments. Moreover, we investigated by NMR the water rebalancing between cell compartments after water stress.

The two-dimensional $\mathrm{T}_{1}-\mathrm{T}_{2}$ correlation measurements, and the comparison of the amplitude of the NMR signals from the distilled water and the outer pericarp before and after water stress, showed clearly that all components corresponded to the water in different cell compartments, and that no signal from non-exchangeable molecules was observed.

The NMR study of the response of specific cell compartments to water stress showed that the vacuole represented the water pool for other cell compartments and that it exchanged water principally with components probably corresponding to the cytoplasm and cell wall. This experiment demonstrated that the NMR relaxation technique is suitable for the continuous monitoring of water rebalancing between subcellular compartments of plant tissues. The method is promising for use in environment-stressed plant research, although further studies are required to evaluate it for physiological changes of water content.

The results of this study represent an important step towards further studies on NMR signal analysis, although more work remains to be done to understand the origin of the signal component with the lowest relaxation time and its accurate assignment to a cell compartment. Further investigations have begun in which the NMR signal of the intact tissue is being compared to various tissue extracts. 
Acknowledgment: The authors wish to thank CTIFL for their collaboration, especially Mr. Letard. We also thank Miss Drouet for her contribution to the experimental work.

\section{REFERENCES :}

1. Hills, B. P., Duce, S. L., (1990). The influence of chemical and diffusive exchange on water proton transverse relaxation in plant tissues, Magnetic Resonance Imaging. 8(3), 321-331.

2. Snaar, J. E. M., Van As, H., (1992). Probing water compartements and membrane permeability in plant cells by H NMR relaxation measurements, Biophys. J. 63, 16541658.

3. Tu, S. Y. S., Choi, Y. J., McCarthy, M. J., McCarthy, K. L., (2007). Tomato quality evaluation by component force and NMR spin-spin relaxation time, Postharvest Biology and Technology. 44(2), 157-164.

4. Duval, F., Cambert, M., Mariette, F., (2005). NMR study of tomato pericarp tissue by spin-spin relaxation and water self-diffusion, Appl Magn Reson. 28(1-2), 29-40.

5. Marigheto, N. A., Moates, G. K., Furfaro, M. E., Waldron, K. W., Hills, B. P., (2009). Characterisation of Ripening and Pressure-Induced Changes in Tomato Pericarp Using NMR Relaxometry, Appl Magn Reson. 36(1), 35-47.

6. Musse, M., Quellec, S., Devaux, M.-F., Cambert, M., Lahaye, M., Mariette, F., (2009). An investigation of the structural aspects of the tomato fruit by means of quantitative nuclear magnetic resonance imaging, Magn Reson Imaging. 27(5), 709719.

7. Musse, M., Quellec, S., Cambert, M., Devaux, M.-F., Lahaye, M., Mariette, F., (2009). Monitoring the postharvest ripening of tomato fruit using quantitative MRI and NMR relaxometry, Postharvest Biol Technol. 53(1-2), 22-35. 
8. Hills, B. P., Remigereau, B., (1997). NMR studies of changes in subcellular water compartmentation in parenchyma apple tissue during drying and freezing, International Journal of Food Science and Technology. 32(1), 51-61.

9. Marigheto, N., Venturi, L., Hills, B., (2008). Two-dimensional NMR relaxation studies of apple quality, Postharvest Biology and Technology. 48(3), 331-340.

10. Sibgatullin, T. A., Anisimov, A. V., de Jager, P. A., Vergeldt, F. J., Gerkema, E., Van As, H., (2007). Analysis of diffusion and relaxation behavior of water in apple parenchymal cells, Biofizika. 52(2), 268-276.

11. Mariette, F., Brannelec, C., Vitrac, O., Bohuon, P. Effet du procédé de friture sur la répartition et l'état de l'eau mesurée par RMN et IRM. in Les produits alimentaires et l'eau, Agoral 99. 1999. Nantes: Edition Tec \& Doc.

12. Furfaro, M. E., Marigheto, N., Moates, G. K., Cross, K., Parker, M. L., Waldron, K. W., Hills, B. P., (2009). Multidimensional NMR cross-correlation relaxation study of carrot phloem and xylem. Part I: Component assignment, Appl Magn Reson. 35(4), 521-535.

13. Raffo, A., Gianferri, R., Barbieri, R., Brosio, E., (2005). Ripening of banana fruit monitored by water relaxation and diffusion H-1-NMR measurements, Food Chem. 89(1), 149-158.

14. Iwaya-Inoue, M., Matsui, R., Sultana, N., Saitou, K., Sakaguchi, K., Fukuyama, M., (2004). H-1-NMR method enables early identification of degeneration in the quality of sweet potato tubers, Journal of Agronomy and Crop Science. 190(1), 65-72.

15. Capitani, D., Brilli, F., Mannina, L., Proietti, N., Loreto, F., (2009). In Situ Investigation of Leaf Water Status by Portable Unilateral Nuclear Magnetic Resonance, Plant Physiology. 149(4), 1638-1647. 
16. Godefroy, S., Callaghan, P. T., (2003). 2D relaxation/diffusion correlations in porous media, Magnetic Resonance Imaging. 21(3-4), 381-383.

17. Mariette, F., Guillement, J. P., Tellier, C., Marchal, P., Continuous relaxation time distribution decomposition by MEM, in Signal Treatment and Signal Analysis in NMR, D.N. Rutledge, Editor. 1996, Elsevier: Paris. p. 218-234.

18. Hopkins, W. G., (2003). Physiologie végétale. Jonh Wiley \$ Sons, Inc., De Boeck Université.

19. Hurlimann, M. D., Burcaw, L., Song, Y. Q., (2006). Quantitative characterization of food products by two-dimensional D-T-2 and T-1-T-2 distribution functions in a static gradient, Journal of Colloid and Interface Science. 297(1), 303-311.

20. Van der Weerd, L., Claessens, M., Van As, H., (2002). Nuclear magnetic resonance imaging of membrane permeability changes in plants during osmotic stress, Plant Cell Environ. 25, 1539-1549.

\section{LEGENDS:}

Fig. 1 :Transverse (a) and longitudinal (b) relaxation time distributions (MEM) for a tomato pericarp sample.

Fig. 2 : Four transverse relaxation time components for a tomato pericarp sample according to saturation-recovery time $\left(\mathrm{t}_{\mathrm{SR}}\right)$ of the $\mathrm{SR}-\mathrm{CPMG}$ sequence $(\bullet-$ component $1, \boldsymbol{\Delta}-$ component 2, - component 3 and $\bullet$ component 4$)$ in $\log / \log$ scale. The $T_{2}$ values were obtained by fitting the individual $T_{2}$ relaxation curves for all $t_{S R}$ values with intensity and relaxation time as adjustment parameters. 
Fig. 3 : SR-CPMG signal decomposed according to $T_{2}$ distribution ( $\bullet-$ component 1 , component 2, $\mathbf{-}$ - component 3 and - component 4 ) according to $t_{S R} . T_{1}$ was calculated for each component by fitting with the mono-exponential model.

Fig. $4: T_{1}-T_{2}$ correlation spectrum for the tomato pericarp. The solid line corresponds to the linear fit of the experimental data and the dashed line corresponds to $T_{1}=T_{2}$.

Fig. 5 : Variations in the NMR signal intensity (CMPG) for four cell compartments according to time before and after application of water stress for the green (a) and red tomatoes (b).

Fig. 6 : Variation in transverse relaxation times (CMPG) for four cell compartments (log scale) according to time before and after applying water stress for the green (a) and red tomatoes (b).

Table 1 : Results from the $T_{2}(C P M G), T_{1}(S R)$ and $T_{1}-T_{2}$ correlation measurements (SRCPMG) on the tomato pericarp sample obtained by the discrete adjustment method. Mean values and standard deviations result from five individual measurements.

Table 2 : Amplitudes of the signals from the fresh and dried tomatoes were compared with the expected signals from the pure water at the same mass and water content as the tomato samples. The signals were normalised by the mean sample masses.

Table 3 : Relative signal intensifies for four cell compartments before applying water stress (i), immediately after (ii) and three hours later (iii) for the green and red tomatoes. 
Fig. 1
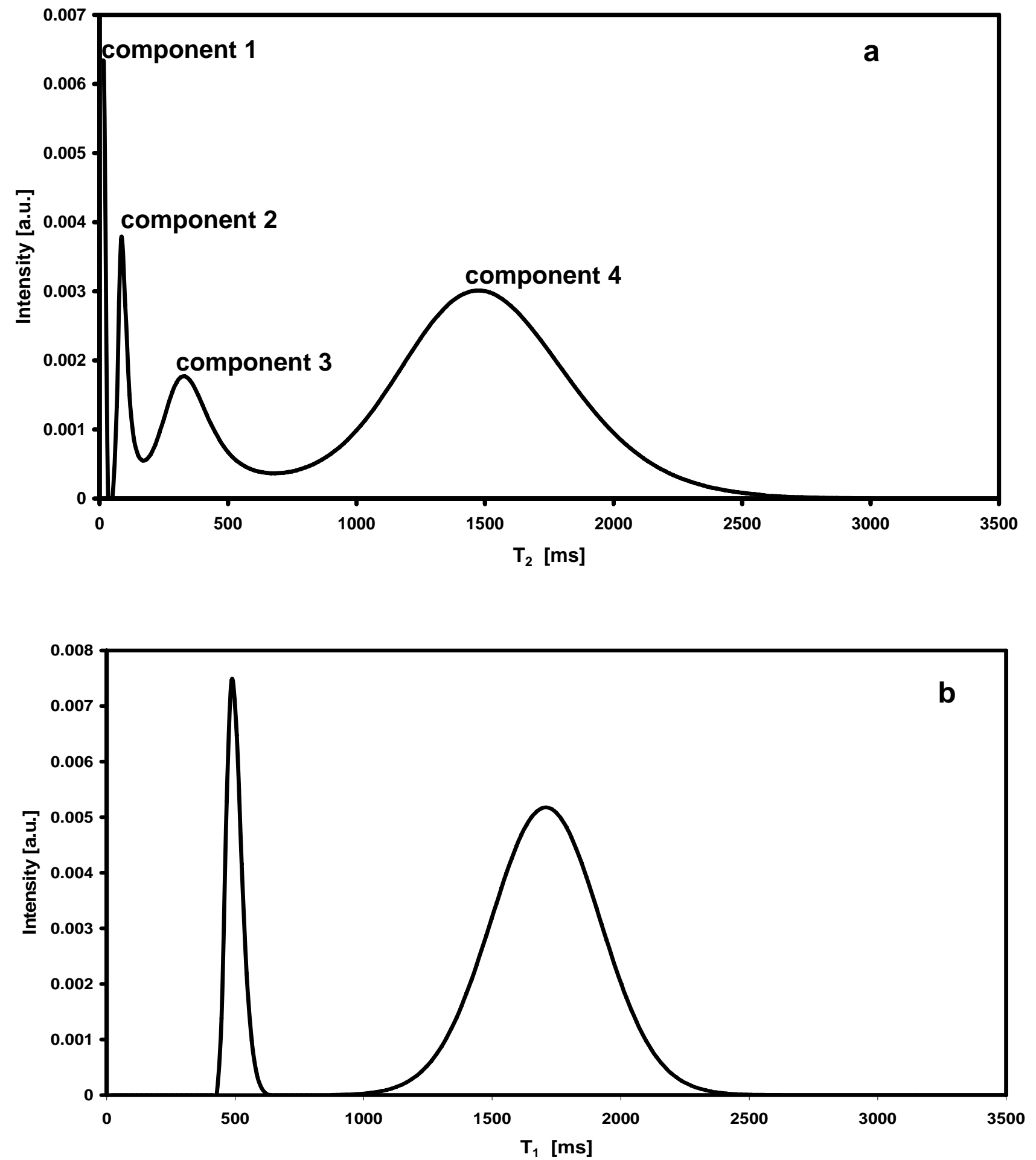


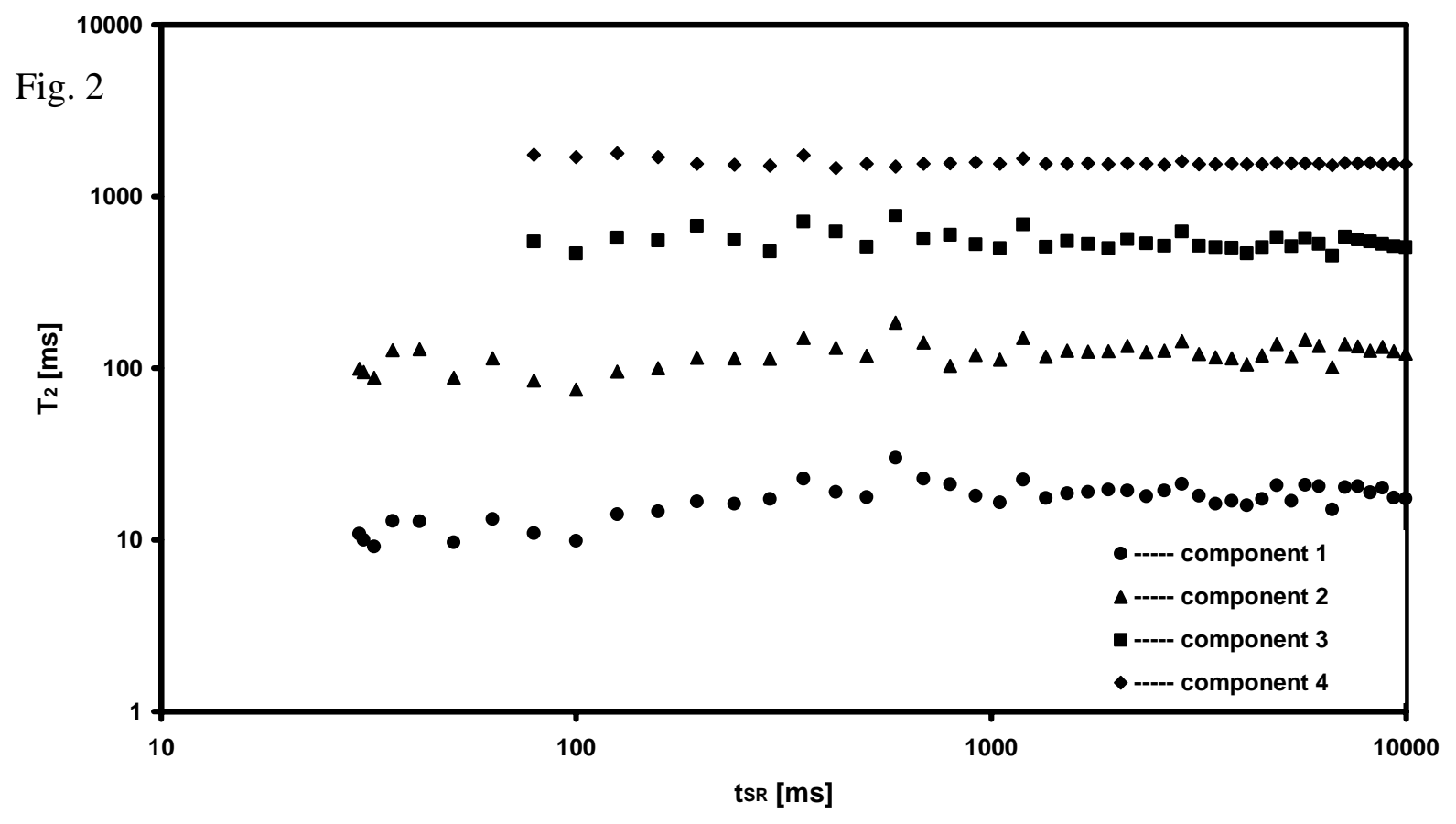




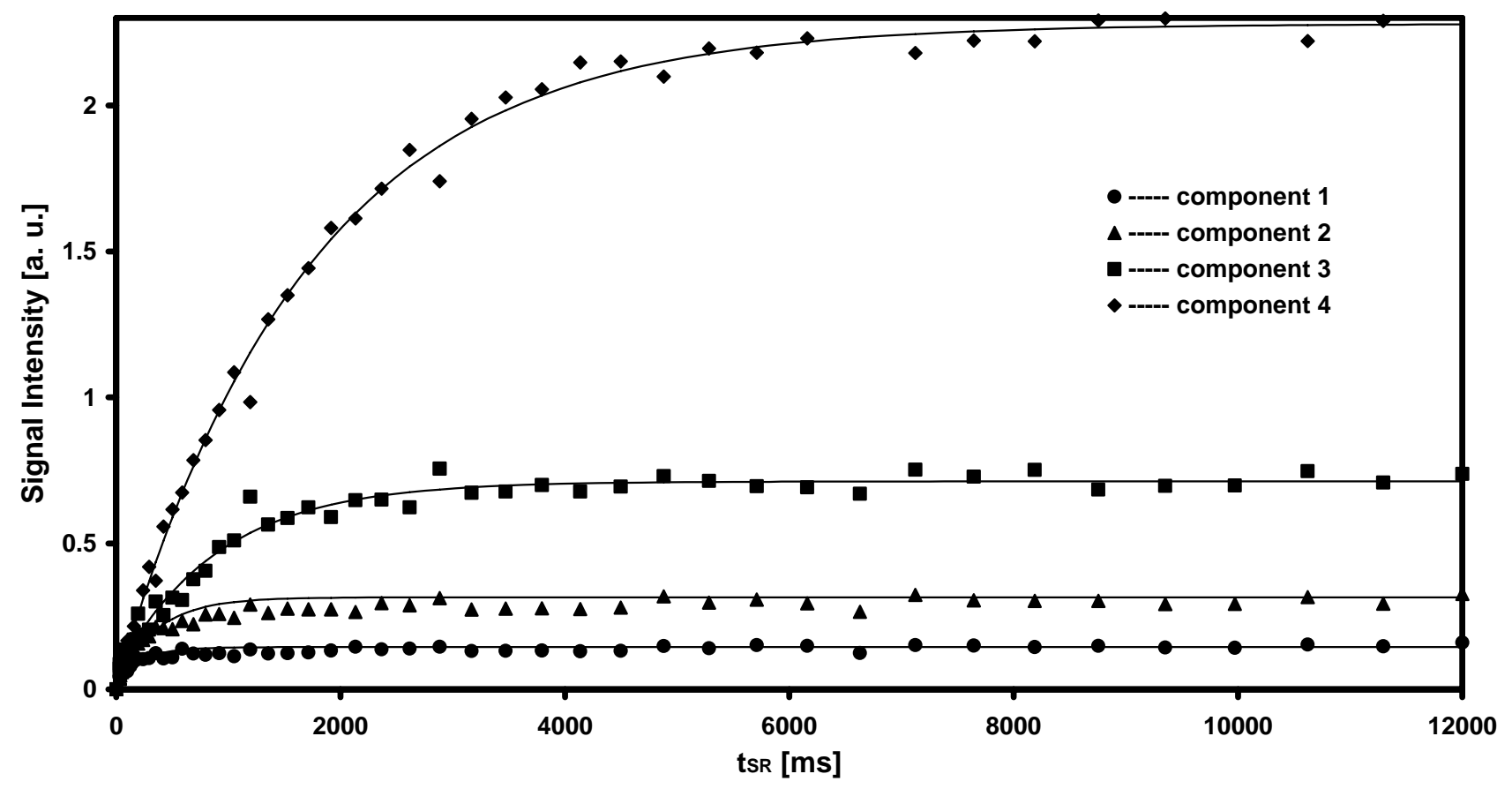

Fig. 3 
Fig. 4

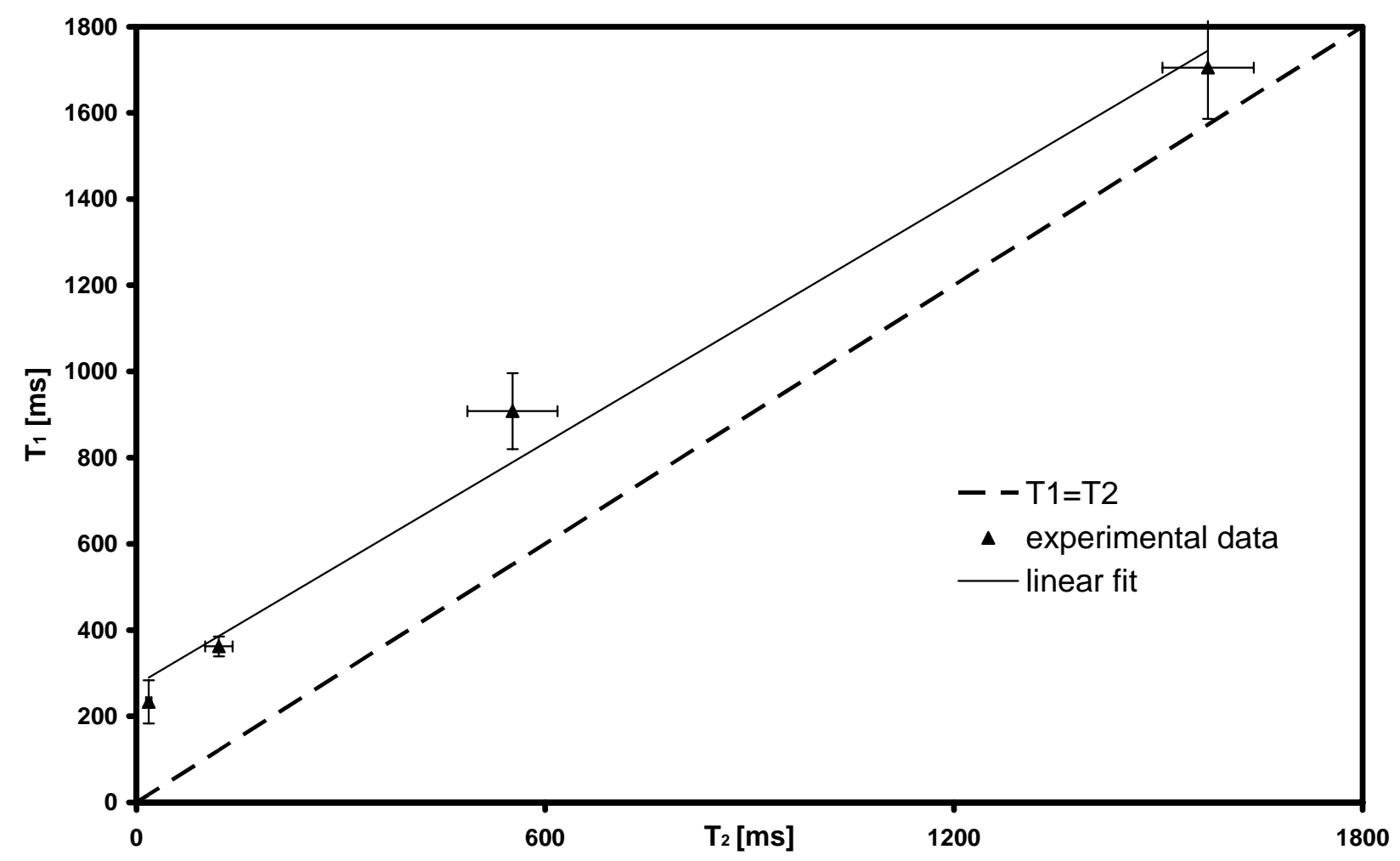


Fig. 5
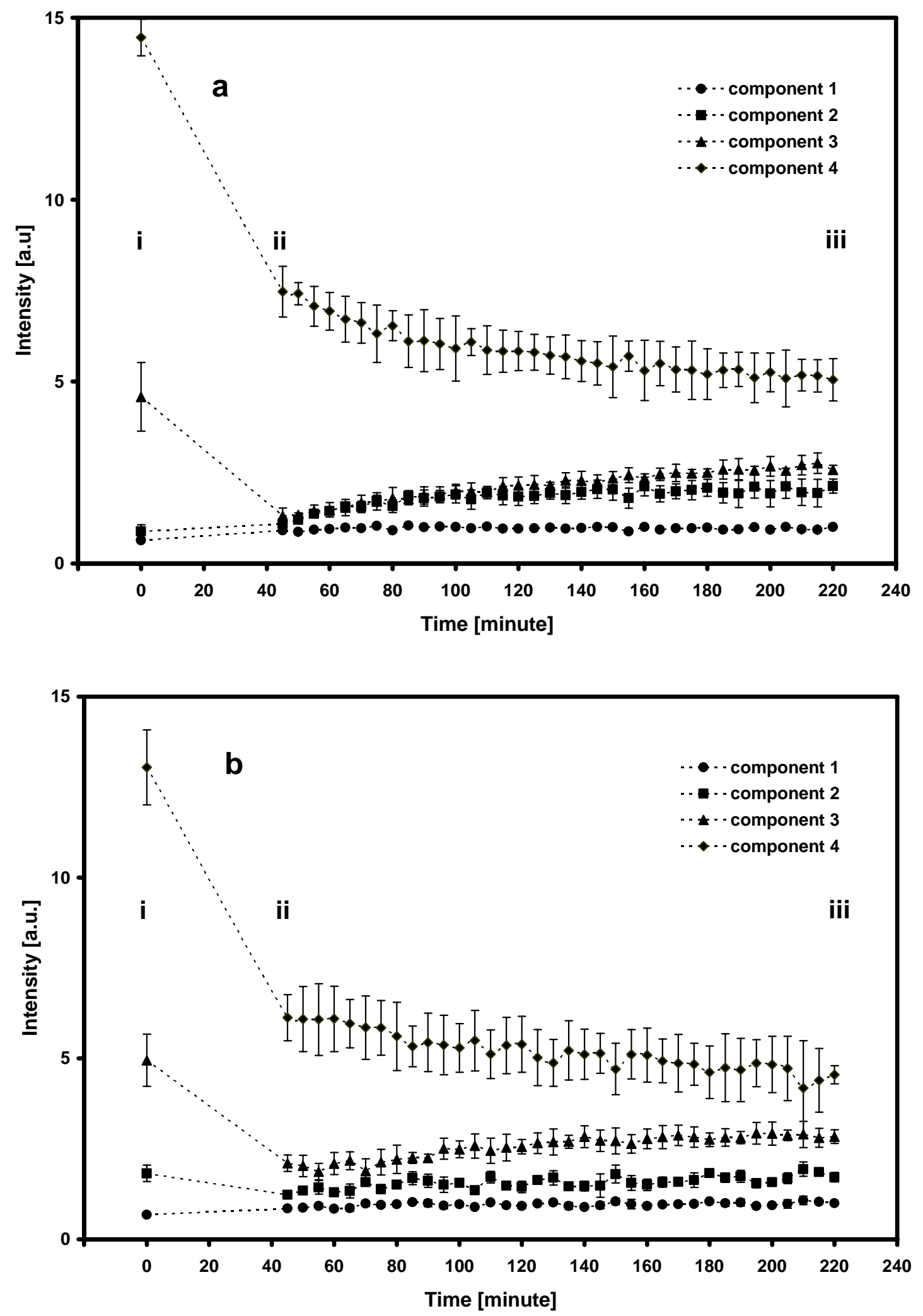
Fig. 6
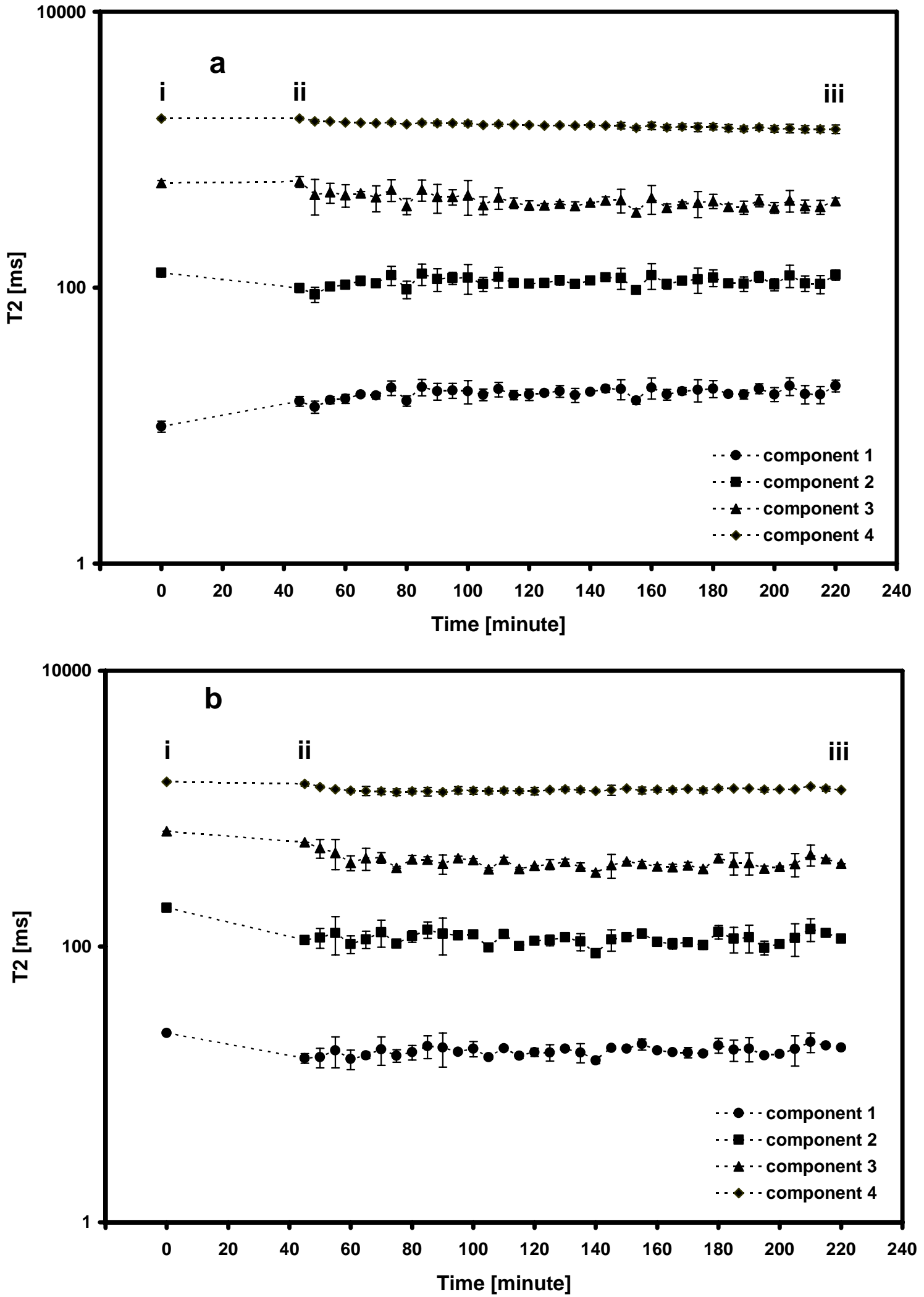


\begin{tabular}{l|c|c|c|c|c|c|c} 
Tab. 1 & \multicolumn{4}{|c|}{1 D } & \multicolumn{3}{c}{ 2 D } \\
& \multicolumn{3}{|c|}{$\mathrm{T}_{2}$ (CPMG) } & \multicolumn{2}{|c|}{$\mathrm{T}_{1}(\mathrm{SR})$} & \multicolumn{3}{c}{$\mathrm{T}_{2}-\mathrm{T}_{1}$ (SR-CPMG) } \\
\hline & $\mathrm{I}[\%]$ & $\mathrm{T}_{2}[\mathrm{~ms}]$ & $\mathrm{I}[\%]$ & $\mathrm{T}_{1}[\mathrm{~ms}]$ & $\mathrm{I}[\%]$ & $\mathrm{T}_{2}[\mathrm{~ms}]$ & $\mathrm{T}_{1}[\mathrm{~ms}]$ \\
\hline Component 1 & $4 \pm 1$ & $18 \pm 4$ & $18 \pm 4$ & $455 \pm 66$ & $7 \pm 4$ & $18 \pm 4$ & $233 \pm 50$ \\
Component 2 & $9 \pm 1$ & $134 \pm 10$ & $82 \pm 4$ & $1644 \pm 44$ & $13 \pm 5$ & $121 \pm 20$ & $362 \pm 23$ \\
Component 3 & $20 \pm 3$ & $551 \pm 49$ & & & $24 \pm 4$ & $552 \pm 66$ & $908 \pm 88$ \\
Component 4 & $68 \pm 5$ & $1528 \pm 62$ & & & $56 \pm 11$ & $1573 \pm 67$ & $1705 \pm 119$
\end{tabular}




\begin{tabular}{l|c|c|c}
\multicolumn{1}{l|}{ Tab. 2 } & Water content [\%] & $\begin{array}{c}\text { Measured signal } \\
\text { intensity ( } \mathrm{I}_{\mathrm{E}} \text { [a.u.] }\end{array}$ & $\begin{array}{c}\text { Expected signal } \\
\text { intensity ( } \mathrm{I}_{\mathrm{T}} \text { [a.u.] }\end{array}$ \\
\hline Fresh tomato & $95.2 \pm 0.0$ & $3.94 \pm 0.01$ & $3.91 \pm 0.01$ \\
\hline Dried tomato & $90.6 \pm 0.1$ & $2.08 \pm 0.01$ & $2.03 \pm 0.01$
\end{tabular}


Tab. 3

\begin{tabular}{l|c|c|c|c|c|c|c|c} 
& \multicolumn{5}{|c|}{ Green tomato } & \multicolumn{4}{c}{ Red tomato } \\
\hline & $\mathrm{I}_{21}[\%]$ & $\mathrm{I}_{22}[\%]$ & $\mathrm{I}_{23}[\%]$ & $\mathrm{I}_{24}[\%]$ & $\mathrm{I}_{21}[\%]$ & $\mathrm{I}_{22}[\%]$ & $\mathrm{I}_{23}[\%]$ & $\mathrm{I}_{24}[\%]$ \\
\hline Point i & $3 \pm 0$ & $4 \pm 1$ & $22 \pm 5$ & $70 \pm 5$ & $3 \pm 0$ & $9 \pm 1$ & $24 \pm 4$ & $64 \pm 5$ \\
Point ii & $9 \pm 0$ & $10 \pm 2$ & $12 \pm 2$ & $69 \pm 4$ & $8 \pm 0$ & $12 \pm 1$ & $21 \pm 3$ & $59 \pm 4$ \\
Point iii & $9 \pm 0$ & $20 \pm 2$ & $24 \pm 2$ & $47 \pm 4$ & $10 \pm 0$ & $16 \pm 2$ & $28 \pm 4$ & $46 \pm 3$
\end{tabular}

